First, we should clarify a specific terminological point, as we used the designation "universal" for those precautions that should be applied to all hemodialysis patients, regardless of their hepatitis B status. This may have been confusing, because the terms "universal" and "standard" currently are used to indicate precautions that apply more generally to all healthcare workers and their contacts with potentially infectious materials. The designation "routine hemodialysis precautions" appears well suited to indicate the approach that is to be followed in any hemodialysis procedure, and we thank Lyerla et al for their appropriate comment on this point.

The next issue raised in their response involves the actual content of these routine hemodialysis precautions. Lyerla et al present a fairly extensive account of these precautions, providing more detail than included in our report. Although some details are subject to discussion, we generally agree that this description represents what should be routine practice in all hemodialysis centers. The use of modern disposable membranes could be included more explicitly, in our opinion. Lyerla et al state that these precautions should be considered sufficient to prevent transmission of bloodborne agents, and our case serves as an illustration of this principle. As an additional requirement, they formulate the condition that these practices must be carried out routinely and rigorously, and subsequently they seriously doubt the feasibility in many hemodialysis centers. If we understand this argument well, they claim that the special precautions for hepatitis B still are needed to compensate for this expected negligence of recommended precautions.

It is somewhat surprising to notice that failures to adhere to rational precautions should thus lead to the maintenance of the exceptional status for hepatitis B in hemodialysis. We are afraid this reasoning contains elements that could lead to the perpetuation of any other wise unnecessary preventive measure. We suggest that risk assessment should be based on the assumption of good practice, as required in these days of protocol-driven medicine and quality control in health care. We are not convinced by the claims that any breach that occurs is much more serious in the case of hepatitis B than, for the sake of comparison, with hepatitis $\mathrm{C}$.

Hepatitis C is more prevalent, leads more often to chronic infection and serious liver damage, and is not preventable by vaccine among patients or staff. Acute infections usually are inconspicuous, masking its occurrence even more. The suggested increased infectivity of hepatitis B is based on incidental high viral loads, not on mean viral titers in carriers, and should not be considered a qualitative difference.

We believe serious inconsistencies exist in the common infection control precautions in hemodialysis, which for some 30 years have focused exclusively on hepatitis B. We learned from reactions to our report that, in The Netherlands, this practice gradually is changing toward a policy of universally applied precautions, the use of which for several years now in one center having three HBV carriers has not lead to transmission. Nevertheless, as put forward by Lyerla et al, this approach relies on the ability of the hemodialysis centers involved to maintain their standards. These centers should, in contact with responsible authorities, decide on the optimal policy in this respect.

Aloys C.M. Kroes, $\mathrm{MD}, \mathbf{P h D}$ Leiden University Hospital

Eric F.H. van Bommel, MD, PhD Drechtsteden Hospital, Dordrecht

Jan A.J.W. Kluytmans, MD, PhD Ignatius Hospital, Breda Willem Weimar, MD, PhD University Hospital Rotterdam The Netherlands

\section{Risk of Tuberculosis Transmission in an Adult Day-Care Center}

\section{To the Editor:}

We describe an investigation to determine the risk of tuberculosis (TB) transmission in an adult daycare facility after identification of a case of active TB in one of its participants. Following the report by a hospital-based physician of active pulmonary and laryngeal TB in an 84year-old man, an outreach publichealth team performed a contact interview and contact investigation in the community setting. Because the patient had underlying schizophrenia, it was difficult to obtain an accurate history regarding the duration of his symptoms. However, his major disease manifestation, progressive difficulty speaking, evolved over several months. He was a member of the day care for more than a year.

The investigation took place within 3 weeks of the patient's diagnosis and included his boarding home and the adult day-care center where he spent 5 to 6 hours daily with 50 other adults, most over the age of 65 . Other than being elderly, the individuals attending the day-care center were not known to be part of any group at increased risk for TB infection. Tuberculin skin testing was performed on contacts using 5 TU purified protein derivative (PPD; $0.1 \mathrm{~mL}$ of Tubersol; Connaught, Swiftwater, PA) per test and interpreted 48 to 72 hours after placement. A positive PPD was defined as $\geqslant 5 \mathrm{~mm}$ of induration. All contacts who had a positive skin test or symptoms of active TB were referred to their personal physicians for chest radiograph. Written recommendations for preventive therapy were provided to the individual care providers.

The boarding-home contact investigation identified eight persons as being at risk for TB exposure from the index case. Half of these were residents of the home, whose contact was limited mainly to communal meals ( 1 to 2 hours daily). One of these individuals, a 47-year-old male, was found to have a 22-mm PPD, resulting in a reaction rate among residents of $13 \%$.

At the day-care center, clients usually sat at tables without assigned seating, so the source case sat with different groups of people every day. Daily programs included a period of singing in which the index case participated actively. The room had only one window, which was used for light only and was sealed. Staff members who worked in this room varied from day to day and usually did not sit at the tables.

As shown in the Table, of the 50 adults who attended the day-care center, 47 received PPD testing (2 had known prior positive tuberculin reactions, and 1 was hospitalized with an unrelated illness and could not be tested). Results of PPD testing were available on 45 adults, approximately $70 \%$ of whom were greater than 65 years of age. Ten individuals (22\%) were identified as having strongly 
TABLE

Purified Protein Derivative Results: Adult Day-Care Center

\begin{tabular}{lcccccc}
\hline Age, $y$ & $\begin{array}{c}\text { No. In } \\
\text { Day Care }\end{array}$ & $\begin{array}{c}\text { No. } \\
\text { Tested* }\end{array}$ & $\begin{array}{c}\text { No. With } \\
\text { Results }\end{array}$ & $\begin{array}{c}\text { No. With } \\
\text { Positive PPD }\end{array}$ & $\begin{array}{c}\text { PPD SIze, } \\
\text { Gender }\end{array}$ & mm \\
\hline$>85$ & 3 & 3 & 3 & 0 & - & - \\
$81-85$ & 3 & 3 & 3 & 0 & - & - \\
$76-80$ & 8 & 8 & 7 & 2 & $2 \mathrm{M}$ & 10,15 \\
$71-75$ & 8 & 6 & 6 & 2 & $\mathrm{M}, \mathrm{F}$ & 20,25 \\
$66-70$ & 13 & 12 & 12 & 2 & M, F & 12,22 \\
$60-65$ & 9 & 9 & 9 & 3 & M, 2 F & $15,20,20$ \\
$<60$ & 6 & 6 & 5 & 1 & F & 15 \\
Total & 50 & 47 & 45 & 10 & $5 \mathrm{M}, 5 \mathrm{~F}$ & \\
\hline
\end{tabular}

Abbreviations: $\mathrm{F}$, female; $\mathrm{M}$, male; $\mathrm{PPD}$, purified protein derivative.

* Two contacts with prior positive PPD and one patient admitted to hospital were not tested.

positive tuberculin tests (range, 10 to $25 \mathrm{~mm}$ ), with 8 having reactions $\geqslant 15$ $\mathrm{mm}$. This unexpectedly high reactor rate, coupled with the extent of induration, implied recent exposure to $\mathrm{TB}$ rather than remote infection. None of these persons had clinical or radiographic evidence of $\mathrm{TB}$. There were no secondary cases of active TB identified. Eleven staff members and 15 drivers also were tested; none were found to be positive.

While there has been an overall decrease in total TB cases in the United States in recent years, certain subgroups have accounted for an increased percentage of reported cases. One such group is foreignborn US residents from countries where TB is endemic; another is the elderly. Senior citizens represent a major reservoir of tuberculous infection, many individuals having become infected with TB early in adulthood during the prechemotherapy era. In 1997 , for example, $23.6 \%$ of all new TB cases were reported in persons over the age of $65 .{ }^{1}$ Reactivation of dormant lesions accounts for approximately 95\% of cases of TB in this group of individuals, ${ }^{2}$ usually as pulmonary disease. However, acquisition of new infection clearly has been documented, particularly in residents of nursing homes and other institutions.

Laryngeal TB is known to exhibit high aerosol infectivity, often arising via bronchogenic extension from a primary pulmonary tuberculous lesion. The presence of both laryngeal and pulmonary lesions in the source case likely was a major factor in the high infectivity rate described in this report. Although the index patient did not manifest prominent cough, aerosolization of droplet nuclei probably was achieved through singing, which carries a similar risk of transmission.

Health department officials utilize the concept of concentric circles when undertaking a contact investigation. The central circle consists of individuals who had the closest and longest duration of contact with the source case. It is important to realize that the center circle is not limited to household members. Because TB is transmitted via the airborne route, individuals who live, work, or are in some way in contact with a source case through a common ventilation system for a prolonged period of time are considered "close contacts" and are at risk of acquiring infection. As such, a thorough contact investigation must include not only the index case's household but often the working and social environment as well.

Recently, the Centers for Disease Control and Prevention published comprehensive guidelines and recommendations to prevent the transmission of $\mathrm{TB}$ in healthcare facilities. 3,4 However, many of the elderly do not reside in such facilities, because they do not require a skilled level of care but rather a low level of supervision. The relatively recent creation of the adult day-care center provides families with the flexibility of having loved ones reside at home, yet have supervision while family members work during the day. Unlike child day-care centers, where much has been written about the spread of infectious diseases, little has been written or is available to address appropriate infection control issues in these new settings.

Although the risk of TB transmission in the elderly population living in closed environments such as nursing homes has been well described, ${ }^{5}$ this outbreak is unique in that it occurred in an adult daycare center. These programs are a relatively recent phenomenon and lack standardized regulations, such as a requirement for baseline tuberculin skin testing. Despite individuals spending much of their day in these settings, adult day-care programs have not been subject to the same scrutiny or infection control measures as nursing homes. With the increasing popularity of congregate settings for senior citizens, testing and surveillance for communicable diseases such as TB become important issues that need to be addressed.

\section{REFERENCES}

1. Centers for Disease Control and Prevention. Reported Tuberculosis in the United States, 1997. Atlanta, GA: CDC; July 1998:16.

2. Dutt AK, Stead WW. Tuberculosis in the elderly. Med Clin North Am 1993;77: 1353-1368.

3. Centers for Disease Control. Prevention and control of tuberculosis in facilities providing long-term care to the elderly. Recommendations of the Advisory Committee for Elimination of Tuberculosis. MMWR 1990;39:7-20.

4. Centers for Disease Control and Prevention. Guidelines for preventing the transmission of Mycobacterium tuberculosis in healthcare facilities. Recommendations and reports. MMWR 1994;43(RR-13):50-54.

5. Stead WW, Lofgren JP, Warren E, Thomas C. Tuberculosis as an endemic and nosocomial infection among the elderly in nursing homes. N Engl J Med 1985;312:1483-1487.

Judith E. Wolf, MD

The Graduate Hospital

James L. Dean, MD

Department of Public Health Philadelphia, Pennsylvania

\section{Level of Suspicion of Pulmonary Tuberculosis Over a 4-Year Period in a Teaching Hospital}

\section{To the Editor:}

The steady decline in the annual rate of tuberculosis (TB) in Canada ended in 1987. Since then, this rate has reached a plateau and varies between 6.9 and 7.4 cases per 100,000 persons per year. The increasing 